Revue internationale P.M.E.

Économie et gestion de la petite et moyenne entreprise

Revure

internationale

PME

\title{
Profil distinctif des dirigeants de PME innovatrices
}

\section{Elisabeth Lefebvre}

Volume 4, numéro 3, 1991

URI : https://id.erudit.org/iderudit/1008068ar

DOI : https://doi.org/10.7202/1008068ar

Aller au sommaire du numéro

\section{Éditeur(s)}

Presses de l’Université du Québec

ISSN

0776-5436 (imprimé)

1918-9699 (numérique)

Découvrir la revue

Citer cet article

Lefebvre, E. (1991). Profil distinctif des dirigeants de PME innovatrices. Revue internationale P.M.E., 4(3), 7-26. https://doi.org/10.7202/1008068ar

\section{Résumé de l'article}

Plusieurs recherches ont démontré l'influence profonde du dirigeant sur l'orientation stratégique de son entreprise et en particulier sur l'adoption d'innovations. Cependant, l'état actuel de nos connaissances ne nous permet pas de cerner avec précision les caractéristiques distinctives du dirigeant ainsi que les modalités par lesquelles s'exerce son influence lors de l'adoption des nouvelles technologies.

Cet article présente les résultats d'une étude empirique qui analyse en profondeur certaines caractéristiques du dirigeant, ce dernier étant considéré comme un facteur primordial d'adoption des nouvelles technologies informatiques dans les PME manufacturières.

Les résultats permettent de constater qu'il existe de fortes relations entre les composantes des profils des dirigeants et le degré innovateur de l'entreprise. En particulier, il ressort clairement qu'une attitude positive envers le risque et une attitude proactive des dirigeants sont les composantes les plus fortement et positivement corrélées au degré innovateur de leurs entreprises. Certaines caractéristiques du processus décisionnel des dirigeants, dont en particulier un horizon de planification orienté vers le long terme, des méthodes d'analyses plus raffinées ainsi qu'une recherche d'informations internes plus systématique sont aussi positivement corrélées au degré innovateur. En outre, les entreprises plus innovatrices bénéficient souvent de l'expérience fonctionnelle plus approfondie de leurs dirigeants en génie-production.

Ces résultats suggèrent qu'au-delà de la simple constatation de l'importance du dirigeant lors de I'adoption des nouvelles technologies, il devient essentiel de connaître les contributions des différentes composantes de ces profils retrouvés dans les entreprises plus innovatrices. Dans une perspective évolutive, le dirigeant d'une PME déterminerait done en grande partie la trajectoire technologique de son entreprise ce qui, sur les plans théorique et pratique, a de profondes répercussions.
Ce document est protégé par la loi sur le droit d'auteur. L'utilisation des services d'Érudit (y compris la reproduction) est assujettie à sa politique d'utilisation que vous pouvez consulter en ligne.

https://apropos.erudit.org/fr/usagers/politique-dutilisation/ 


\title{
Profil distinctif des dirigeants de PME innovatrices
}

\author{
Élisabeth LEFEBVRE \\ École polytechnique de Montréal*
}

\begin{abstract}
RÉSUMÉ
Plusieurs recherches ont démontré l'influence profonde du dirigeant sur l'orientation stratégique de son entreprise et en particulier sur l'adoption d'innovations. Cependant, l'état actuel de nos connaissances ne nous permet pas de cerner avec précision les caractéristiques distinctives du dirigeant ainsi que les modalités par lesquelles s'exerce son influence lors de l'adoption des nouvelles technologies.
\end{abstract}

Cet article présente les résultats d'une étude empirique qui analyse en profondeur certaines caractéristiques du dirigeant, ce dernier étant considéré comme un facteur primordial d'adoption des nouvelles technologies informatiques dans les PME manufacturières.

Les résultats permettent de constater qu'il existe de fortes relations entre les composantes des profils des dirigeants et le degré innovateur de l'entreprise. En particulier, il ressort clairement qu'une attitude positive envers le risque et une attitude proactive des dirigeants sont les composantes les plus fortement et positivement corrélées au degré innovateur de leurs entreprises. Certaines caractéristiques du processus décisionnel des dirigeants, dont en particulier un horizon de planification orienté vers le long terme, des méthodes d'analyses plus raffinées ainsi qu'une recherche d'informations internes plus systématique sont aussi positivement corrélées au degré innovateur. En outre, les entreprises plus innovatrices bénéficient souvent de l'expérience fonctionnelle plus approfondie de leurs dirigeants en génie-production.

Ces résultats suggèrent qu'au-delà de la simple constatation de l'importance du dirigeant lors de l'adoption des nouvelles technologies, il devient essentiel de connaitre les contributions des différentes composantes de ces profils retrouvés dans les entreprises plus innovatrices. Dans une perspective évolutive,

* Élisabeth Lefebvre détient un $\mathrm{Ph}$. D. en administration (option gestion de la technologie). Ses intérêts de recherche actuels portent tout particulièrement sur les différents facteurs susceptibles de promouvoir l'adoption des technologies avancées dans les entreprises. Adresse : École polytechnique, Université de Montréal, Département de génie industriel, C.P. 6079, succursale A, Montréal (Québec), H3C 3A7.

Revue intemationale P.M.E., vol. 4, $\mathrm{n}^{\circ}$ 3, 1991 
le dirigeant d'une PME déterminerait donc en grande partie la trajectoire technologique de son entreprise ce qui, sur les plans théorique et pratique, a de profondes répercussions.

\begin{abstract}
Many researchers have demonstrated the influence of the CEO (Chief executive officer) on the strategic orientation of the firm, particularly concerning the adoption of innovations. Nevertheless, it has proved difficult to pin down the CEO's exact distinctive characteristics as well as the characteristics of his decision making process as it pertains to technology adoption.
\end{abstract}

This paper presents results from an empirical study which analyzes in details some of those CEO characteristics, the underlying assumption being that he is one of the main influential factor in the adoption of new computerized technologies especially in the context of small manufacturing firms.

The results show the existence of strong relationships between the CEO's profile and the innovativeness of the firm. It clearly appears that a positive attitude towards risk and a proactive attitude are the strongest determinants and the most positively correlated to the degree of innovativeness of his firm. Some characteristics of the CEO's decision-making process are also positively related to the innovativeness, namely a long-term oriented planification, greater analytical ability, and a more elaborate systematic internal search for information. Furthermore, the CEOs in the more innovative firms have often a sounded engineering-production experience.

The results also suggest that over and above the basic observation of the CEO's importance in the new technology adoption process, it is crucial to find out all the different profile components implied in the most innovative firms. In an evolutive perspective, the CEO of a small business would be mainly responsible of the technological orientation of his firm which leads to important consequences both on theoretical and practical grounds.

\title{
RESUMEN
}

Varias investigaciónes han demostrado la profunda influencia del dirigente en la orientación estratégica de su empresa, particularmente en las inovaciones. Sin embargo, el estado actual de nuestros conocimientos no nos permite de conseguir precisamente, las caracteristicas distintas del dirigente y los modos por los cuáles se refleja su influencia cuando se adoptan nuevas tecnologias.

El siguiente artículo presenta los resultados de un estudio empirico que analiza con todo detalle ciertas caracteristicas del dirigente, éste, siendo considerado como un prime factor en la adopción de las nuevas tecnologías informáticas de la PME (pequeña y mediana empresa) manufacturera.

Los resultados permiten tener en cuenta que hay importantes relaciones entre los conponentes del perfil de los dirigentes y el nivel innovador de la empresa. En particular, se nota claramente que un actitud positiva frente al riesgo y un actitud pro-activa de los dirigentes son fuertamente y positivamente ligadas al nivel innovador de sus empresas. Ciertas características del proceso decisional 
de los dirigentes, particularmente cuando se trata de una planificación, a largo termino, son tambien positivamente ligadas al nivel innovador como los métodos de análisis mas profundos y una investigación sistemática más intensa de la información interna. Una experiencia funcional mas elevada en la producción de la inginiera se encuentra en las empresas mas innovadoras. Finalmente, dos variables estructurales como la tecnocracia y las estructuras organizacionales más flexibles favorecen las innovaciones.

Esto sugiere que, cuando se adoptan nuevas tecnologias, más importantes que la del dirigente, parece necesario de reconocer la importancia de los otros componentes de esos perfiles encontrados en las empresas innovadoras. En una perspectiva evolutiva, el dirigente de una PME determinaría mayormente la trayectoria tecnológica de su empresa, la cuol, teoricamente y práticamente tiene profundas repercusiones.

\section{Introduction}

Au cours de la dernière décennie nous avons été témoins d'une intensification de la compétition industrielle globale, imposant aux entreprises manufacturières de nouvelles pressions à la compétitivité et des exigences de productivité accrues. Cette tendance devrait même s'accentuer au cours des prochaines années. Dans un tel contexte, l'adoption des nouvelles technologies, en raison des avantages stratégiques qui permettent de consolider et d'améliorer la position compétitive des entreprises, devient une priorité. Or, l'industrie nord-américaine accuse un sérieux retard sur ce plan. De toute évidence, plusieurs facteurs peuvent être invoqués pour expliquer que nos entreprises n'adoptent pas les nouvelles technologies au même rythme que les autres pays industrialisés. Un des facteurs déterminants serait, selon plusieurs auteurs, l'influence des membres de la haute direction.

Pour les PME, l'adoption de nouvelles technologies constitue une décision stratégique qui pourrait leur permettre de maintenir une position concurrentielle actuellement de plus en plus menacée (Lefebvre et al., 1990). Si l'on considère l'important investissement de capitaux et l'exigence d'une maind'œuvre spécialisée, la survie même de l'entreprise peut dépendre de ce choix. Il n'est donc pas étonnant de constater la forte implication des dirigeants dans cette prise de décision. Plusieurs recherches ont fait état de ce rôle dominant et nécessaire des dirigeants de PME pour engendrer l'innovation et assurer la pleine réalisation du potentiel technologique. Cependant, l'état actuel de nos connaissances ne nous permet pas de cerner avec précision les caractéristiques distinctives et le rôle primordial du dirigeant, ainsi que les modalités par lesquelles s'exerce son influence lors de l'adoption de nouvelles technologies. C'est pourquoi nous avons mené une recherche dont l'objectif principal visait à mieux comprendre certaines des caractéristiques des dirigeants associés aux entreprises les plus innovatrices dans l'utilisation des technologies informatiques. 


\section{Le dirigeant et l'adoption des nouvelles technologies dans son entreprise}

L'influence du dirigeant lors de l'adoption de nouvelles technologies est primordiale. Dans le cas des petites entreprises qui ont fait le premier pas vers l'informatisation, le dirigeant est non seulement le premier à avoir l'idée d'introduire les technologies de l'information, mais il est de plus celui qui, dans plus de $90 \%$ des cas, prend la décision d'informatiser l'entreprise (Lefebvre et Lefebvre, 1988b). Même dans les très grandes entreprises et dans le contexte plus général d'adoption des nouvelles technologies, le dirigeant joue un rôle très actif (CEC, 1987 et 1986 ; Maidique et Hayes, 1985).

Toutefois, on retrouve également la situation inverse où l'influence du dirigeant s'avère négative et constitue ainsi l'un des principaux obstacles à l'adoption des nouvelles technologies. Dans le contexte nord-américain, de nombreux chercheurs dénoncent cette situation (Munro et Noori, 1987 ; Skinner, 1985,1984 et 1983 ; Hayes et Wheelwright, 1984, pour n'en nommer que quelques-uns). De même, il semblerait que les attitudes des dirigeants canadiens ne soient pas de nature à promouvoir l'adoption et la diffusion des nouvelles technologies. En se basant sur une enquête comparative entre les États-Unis, le Canada, l'Europe et le Japon, Davis (1985) arrivait à la conclusion que les dirigeants canadiens étaient, en moyenne, moins ouverts aux innovations que leurs collègues japonais. D'autres études comparatives sur l'attitude des dirigeants envers les innovations existent aussi : la plupart d'entre elles emploient une méthodologie qualitative et comparent les dirigeants américains aux dirigeants japonais (Hayes et Wheelwright, 1984 ; Marsland et Beer, 1983 ; Schonberger, 1982 ; Baranson, 1981 ; Hayes, 1981 ; Pascale et Athos, 1981), ou aux dirigeants allemands (Limprecht et Hayes, 1982 ; Lawrence, 1980). Toutes en viennent à la conclusion qu'en Amérique du Nord, la haute direction est moins favorable à l'adoption des nouvelles technologies que dans les autres pays. Ce même problème est soulevé par les praticiens. Ainsi, dans une enquête menée en 1987 auprès des quelque quatre mille lecteurs de la revue américaine Harvard Business Review, l'opinion prédominante était que la piètre performance des gestionnaires se trouve à l'origine du déclin de la compétitivité.

Dans la mesure où tout semble confirmer que, dans certaines situations, le dirigeant est l'un des principaux obstacles à l'adoption des nouvelles technologies, nous étudierons exhaustivement son apport en abordant quatre aspects cruciaux : ses caractéristiques personnelles, ses attitudes et ses traits de personnalité, les particularités de son processus décisionnel, ainsi que les propriétés organisationnelles liées à son influence. 


\subsection{Les caractéristiques personnelles du dirigeant}

Dans le contexte nord-américain, plusieurs caractéristiques émergent de façon particulièrement frappante. Une de ces caractéristiques, la mobilité excessive des hauts gestionnaires américains, a été dénoncée tout particulièrement par Hayes et Abernathy (1980). Cette mobilité des hauts gestionnaires occasionnerait, entre autres, des décisions basées sur des informations inadéquates et incomplètes, et une tendance à ne se préoccuper que du court terme.

L'expérience fonctionnelle, ainsi que le bagage scolaire du dirigeant, la plupart du temps non technique, ne favoriseraient pas l'adoption des nouvelles technologies (Dean, 1987 ; Gerstein et Reisman, 1984 ; Hayes et Wheelwright, 1984 ; Kantrow, 1980). Or, ces deux aspects sont primordiaux puisque, selon McMillan (1987), la priorité pour la gestion des entreprises performantes de demain est de comprendre l'innovation technologique.

Ces caractéristiques personnelles du dirigeant, que nous pourrions appeler fiche signalétique, seront mesurées par les 7 variables de recherche présentées au tableau 1 (premier ensemble de variables indépendantes).

\section{TABLEAU 1}

\section{Variables de recherche retenues et mesures opérationnelles utilisées}

\section{Valeur dépendante}

- adoption d'innovations incrémentales

- adoption d'innovations radicales

\section{Variable indépendante}

i) Caractéristiques de l'entreprise

V1 : propriétaire de l'entreprise

V2: nombre d'années de service dans l'entreprise

V3 : nombre d'années de service dans ce secteur d'activité

V4: nombre d'années de service dans sa fonction de dirigeant

V5: expérience fonctionnelle

V6: formation technique

V7: niveau d'éducation
Mesure utilisée par Dewar et Dutton, 1986

\section{Mesure utilisée par Kahn et} Manopichetwattana, 1989

Mesure utilisée par Jarymiszyn et al., 1985

Mesure utilisée par Collins et al., 1988

Mesure utilisée par Julien et al., 1988

Mesure utilisée par Julien et al., 1988

ii) Attitudes et traits de personnalité du dirigeant

V8: attitude envers les changements technologiques

V9: attitude envers le risque
Mesure utilisée par Julien et al., 1988 et Nabseth, 1973

Mesure adaptée de Kahn et Manopichetwattana, 1989 
V10: attitude proactive

V11 : champ de contrôle (locus of control)

iii) Caractéristiques du processus décisionnel du dirigeant

V12: recherche systématique d'information interne

V13 : recherche systématique d'information externe

V14 : horizon de planification

V15: analyse

V16: conscience de la stratégie

iv) Caractéristiques organisationnelles liées à l'influence du dirigeant

V17 : technocratisation

V18 : centralisation

V19 : formalisation
Mesure utilisée par Lumpkin, 1988

Mesure adaptée de Miller et Friesen, 1982

Mesure adaptée de Kahn et Manopichetwattana, 1989

Mesure utilisée par Collins et al., 1988 et Julien et al., 1988

Mesure utilisée par Cohn et Turyn, 1984 et Hage et Aiken, 1970 et 1967

Mesure utilisée par Cohn et Turyn, 1984

\subsection{Attitudes et tralts de personnalité du dirigeant}

Compte tenu de l'influence du dirigeant dans la gestion et la direction de l'entreprise, et ce, particulièrement dans les PME (D'Amboise, 1989; Julien et Marchesnay, 1988 ; Castaldi, 1986), ses attitudes envers les changements en général et les changements technologiques en particulier sont associées à l'adoption d'innovations (Julien et al., 1988). Parallèlement, une attitude favorable envers le risque et l'innovation des produits ainsi qu'une attitude proactive sont positivement associées à l'adoption d'innovations. Selon Kahn et Manopichetwattana (1989), ces trois aspects mesurent aussi la capacité entrepreneuriale.

Quant aux traits de personnalité du dirigeant, le champ de contrôle (locus of control) a été souvent associé au comportement entrepreneurial (Brockhaus, 1982 et 1975 ; Shapero, 1975 ; Durand et Shea, 1974) et a été plus récemment identifié comme un "influenceur » du caractère innovateur des entreprises (Kahn et Manopichetwattana, 1989 ; Miller et Toulouse, 1986a et 1986b ; Miller, Kets de Vries et Toulouse, 1982). Le champ de contrôle (Rotter, 1966) reflète la perception qu'a un individu du contrôle qu'il est en mesure d'exercer sur les événements qui peuvent lui arriver. Ainsi, une personne dont le champ de contrôle est interne serait convaincue que ses efforts ont permis de changer le déroulement des événements, tandis qu'une personne dont le champ de contrôle 
est externe serait persuadée que le destin, la fatalité ou la chance influencent le déroulement des événements. Ainsi, il existerait une association positive entre le champ de contrôle interne et l'innovation (Kahn et Manopichetwattana, 1989; Miller et Toulouse, 1986a et 1986b).

\subsection{Le processus décisionnel du dirigeant}

La façon même dont la décision d'adopter les nouvelles technologies est prise deviendrait, selon plusieurs auteurs, l'un des facteurs d'adoption les plus influents (Dean, 1987 ; Meyer et Goes, 1987 ; Pennings, 1987). Puisque le dirigeant a une influence considérable lors de l'adoption des nouvelles technologies, il en découle logiquement que son implication lors des différentes phases du processus décisionnel devient primordiale.

On a donc retenu quatre variables (troisième ensemble de variables indépendantes), correspondant aux différentes phases du processus décisionnel proposé initialement par Simon (1977) et repris plus récemment par Meyer et Goes (1987). Ainsi, à la phase « intelligence " (ou recherche d'informations) correspondrait la recherche systématique d'informations internes ou externes à l'entreprise (scanning). Les trois autres variables, l'horizon de planification (futurity), la prise de conscience et l'analyse de la stratégie (consciousness of strategy) se rapportent à la phase « évaluation-choix ».

\subsection{Les caractéristiques organisationnelles liées à l'influence du dirigeant}

Les caractéristiques organisationnelles (quatrième ensemble de variables indépendantes) présentant le plus d'intérêt pour notre étude sont celles qui sont fortement influencées par le dirigeant de l'entreprise et qui peuvent ultimement promouvoir l'adoption des nouvelles technologies.

L'influence du dirigeant sur la structure organisationnelle de son entreprise est bien reconnue dans la littérature, surtout lorsqu'il s'agit de petites entreprises (D’Amboise, 1989 ; Julien et Marchesnay, 1988). Certains auteurs sont même allés plus loin en démontrant empiriquement la relation entre certains traits de personnalité du dirigeant et certaines configurations organisationnelles (Miller et Toulouse, 1986a ; Miller et Dröge, 1986 ; Kets de Vries et Miller, 1984).

Plusieurs auteurs ont aussi démontré l'importance de certaines caractéristiques organisationnelles particulières quant à la capacité innovatrice des entreprises. Ainsi, la technocratisation, c'est-à-dire le degré de connaissances techniques et scientifiques retrouvées dans une entreprise, serait la caractéristique organisationnelle la plus souvent associee à l'adoption d'innovations tech- 
nologiques (Collins et al., 1988; Julien et al., 1988). En ce qui concerne la relation entre la technocratisation organisationnelle et certains traits spécifiques du dirigeant, on pourrait croire intuitivement à une complicité relativement étroite, bien qu'elle ne soit que partiellement démontrée.

Deux autres propriétés organisationnelles associées à certains traits du dirigeant, soit la formalisation et la centralisation, semblent pour leur part être négativement liées au caractère innovateur des entreprises (Cohn et Turyn, 1984 ; Hage et Aiken, 1970 et 1967). En effet, une décentralisation stimule la créativité et l'innovation, bien qu'il soit possible de soutenir l'argument inverse : une plus grande centralisation peut aussi faciliter la décision d'adopter des innovations en limitant, par exemple, le nombre d'intervenants (Zaltman et al., 1973 ; Normann, 1971 ; Rogers et Shoemaker, 1971).

\section{Aspects méthodologiques}

Un certain nombre de difficultés semblent être inhérentes à la recherche en innovation, car les résultats ont été qualifiés d'instables, peu convergents, non interprétables, voire contradictoires (Meyer et Goes, 1987 ; Pennings, 1987 ; Ettlie et al., 1984 ; Downs et Mohr, 1976).

\subsection{Difficultés méthodologiques reliées au choix de la variable dépendante}

Une première difficulté est étroitement liée à la pluralité des définitions du terme « innovation ». Or, compte tenu du fait que l'innovation constitue la variable dépendante dans la majorité des recherches, les problèmes crés par la difficulté de saisir les attributs de l'innovation engendrent, selon plusieurs auteurs, des points de vue à la fois fragmentaires et contradictoires (Dewar et Dutton, 1986). Ainsi, selon Downs et Mohr, il existerait deux niveaux d'attributs pour déterminer les types d'innovations : des attributs dits primaires, qui se rapportent à l'objet (l'innovation) et qui sont indépendants du sujet (l'organisation), et des attributs dits secondaires, qui varient selon la perception que les sujets ont de l'objet. C'est ainsi que certains attributs d'une innovation peuvent être les mêmes pour toutes les organisations, alors que d'autres varient selon le type d'organisation concernée. Or, selon ces mêmes auteurs, les attributs secondaires sont les plus susceptibles d'indiquer le caractère innovateur d'une organisation. Cependant, dans la majorité des recherches, les attributs primaires et les attributs secondaires ne sont pas départagés, ce qui conduit, dans plusieurs cas, à des résultats non interprétables, puisque l'on ne peut distinguer l'apport d'un déterminant donné à un type particulier d'innovation dans une organisation spécifique. 
Pour pallier les difficultés méthodologiques reliées au choix de la variable dépendante, nous devons déterminer l'innovation aussi précisément que possible, puisque des différences fondamentales existent entre différents types d'innovation. Nous avons donc retenu des innovations qui partagent le même attribut primaire ; ces innovations correspondent toutes à des applications de technologies informatiques (tableau 2). La typologie utilisée est dérivée de recherches empiriques (Statistique Canada, 1988 et 1989; Lefebvre et Lefebvre, 1988a) elle comprend à la fois les applications associées aux technologies de bureau et aux technologies de production. Ces deux dernières technologies étant de plus en plus intégrées, particulièrement dans le secteur manufacturier, il devient impossible de les dissocier (Goldhar et Jelinek, 1985, Child et al., 1983).

TABLEAU 2

Typologie d'innovations de procédés informatisés

Applications associées aux technologies de bureau

- Comptes à payer/comptes à recevoir

- Gestion des inventaires

- Analyse des ventes

- Gestion de la paie

- Facturation

- Prix de revient

- Gestion des opérations

- Traitement de texte

- Courrier/archivage électronique

Applications associées aux technologies de production

- Conception et ingénierie

- conception assistée par ordinateur (CAO) et ingénierie assistée par ordinateur (IAO)

- résultats CAO servant au contrôle des machines de fabrication CAO/FAO

- Fabrication et assemblage

- machines à commandes numériques informatisées

- cellules de fabrication flexible (CFF)

- systèmes de fabrication flexible (SFF)

- robots de mise en place simples

- autres robots plus complexes

- Manipulation automatisée du matériel

- système de stockage et d'extraction automatisé (SSEA)

- système de véhicules guidés automatiques (SVGA)

- Inspection par capteurs automatisés et/ou vérification d'équipement

- Communications et contrôle

- réseau local pour l'usine

- réseau informatique entre compagnies reliant les usines aux fournisseurs et aux clients

Source : Statistique Canada, (1989 et 1988); Lefebvre et Lefebvre, (1988a). 
Quant à l'attribut secondaire de ces innovations, nous avons choisi le critère récemment proposé par Dewar et Dutton (1986) et Ettlie et al. (1984) dans le cadre de recherches empiriques. Selon ces derniers, une innovation serait de nature radicale ou incrémentale, selon les contextes organisationnels et industriels considérés.

\subsection{Définition de la variable dépendante}

Pour déterminer de façon objective la nature incrémentale ou radicale de chacune des innovations considérées, nous avons fait appel à un panel de 20 experts venant de divers milieux (universitaire, public, parapublic et privé) familiers avec les procédés informatisés généralement retrouvés dans les entreprises du secteur considéré. L'information obtenue nous a permis de classer les technologies informatisées selon un continuum « incrémental-radical » puis par la suite, d'établir un groupe d'innovations radicales et un groupe d'innovations incrémentales.

Les résultats obtenus auprès du panel d'experts illustrent bien l'écart entre les applications technologiques de base, telles la gestion de la paie, et les applications plus sophistiquées, telles que le CAO/IAO. Les applications associées aux technologies de bureau, sauf la gestion des inventaires, du prix de revient et la gestion des opérations, sont généralement considérées par les experts comme des innovations de nature plus incrémentale. Ces trois dernières opérations se distinguent des applications comptables de base (gestion de la paie, comptes à payer/a recevoir, facturation) et des applications de nature opérationnelle (traitement de texte): elles impliquent une certaine préoccupation envers le contrôle des activités de l'entreprise et, en ce sens, constituent une indication d'une grande maturité technologique (Gibson et Nolan, 1974).

La plupart des innovations qualifiées de plus radicales par les experts correspondent à des applications associées aux technologies de production. Le fait que les machines à commandes numériques informatisées soient considérées parmi les innovations radicales comme celles qui le seraient le moins semble relativement raisonnable. Elles constituent en effet une forme d'automatisation assez rudimentaire et courante (MIC, 1987 ; CEC, 1986). La conception assistée par ordinateur et/ou l'ingénierie assistée par ordinateur atteint le plus haut niveau dans les innovations radicales. Le savoir-faire technologique nécessaire à la mise en place et à l'exploitation de tels systèmes justifie en effet le classement de cette innovation dans la catégorie radicale. Il faut aussi remarquer que les technologies de télécommunication associées à l'automatisation des activités de production (réseau local pour l'usine et réseau informatique entre compagnies reliant les usines aux fournisseurs et aux clients) sont aussi évaluées par les experts comme des innovations radicales, compte tenu de la taille restreinte des entreprises considérées. 
Évaluer le degré innovateur de façon dichotomique (adoption d'innovations incrémentales contre adoption d'innovations radicales) apparaît comme une simplification légèrement abusive. En effet, nous pensons que, dans un contexte de PME, l'adoption de plusieurs innovations incrémentales peut représenter un pas plus décisif et une avance technologique plus considérable que l'adoption d'une seule innovation radicale. Il s'agirait dès lors de nous éloigner de cette simple dichotomie pour tenter de redéfinir le degré innovateur d'une entreprise.

La typologie des innovations considérées comprend 21 applications informatisées pour lesquelles le panel d'experts a accordé un score sur une échelle variant entre 1 (= incrémental) et 7 (= radical). Il est donc proposé de pondérer chaque innovation adoptée par le score moyen accordé par les vingt experts et de faire l'addition de toutes les innovations adoptées ainsi pondérées. Cette somme pondérée donne non seulement le nombre d'innovations (comme mentionné dans des études précédentes ; voir, par exemple, Lefebvre et al., 1990; Ettlie et al., 1984 ), mais aussi la nature radicale et incrémentale de ces innovations. Cette façon de quantifier le degré innovateur est inspirée du score d'automatisation proposé par Khandwalla (1977). Cependant, Khandwalla pondère selon des critères prétablis qui ne tiennent pas compte du milieu organisationnel et sectoriel. Le degré innovateur ainsi calculé représente la variable dépendante retenue dans le cadre de cette recherche.

\subsection{Cholx du contexte organisationnel}

Plusieurs recherches ont démontré l'importance des caractéristiques organisationnelles lors de l'adoption d'innovations. Pensons tout particulièrement à la taille de l'entreprise (Miller et Toulouse, 1986 ; Lefebvre et al., 1985) et au secteur d'activités. Le retranchement de certaines caractéristiques organisationnelles pourrait expliquer partiellement pourquoi certains résultats de recherche sont si contradictoires. Pour pallier cette difficulté, l'étude présentée ici est restreinte à un groupe d'entreprises de même taille et de même secteur d'activités.

L'univers visé par l'enquête est très clairement défini : il s'agit d'entreprises manufacturières établies au Québec dans le secteur d'activités appelé produits en matières plastiques et dont la taille varie entre 10 et 200 employés. Cette limite inférieure très arbitraire permet d'exclure les très petites entreprises qui ont, dans l'ensemble, très peu adopté d'innovations de procédés. Cette forme d'exclusion a priori a été retenue pour les mêmes raisons dans d'autres recherches (par exemple, Statistique Canada, 1988 ou Raymond, 1987). De plus, ces très petites entreprises encore appelées «entreprises artisanales " ou « hypofirmes » (entreprises de moins de 10 employés, voire de moins de 5 employés) possèdent des caractéristiques particulières qui les distinguent des autres petites 
entreprises (Julien et Marchesnay, 1988) et qui ne présenteraient pas certaines caractéristiques organisationnelles qui nous intéressent particulièrement, comme la technocratisation. La limite supérieure (moins de 200 employés) est celle qui est fixée dans la définition standard des PME (MIC, 1987).

Nous avons choisi, comme secteur spécifique, celui des produits en matières plastiques, puisque les innovations considérées y sont toutes potentiellement applicables. De plus, ce secteur affiche un taux relativement élevé de pénétration de ces innovations (Association CAO/FAO, 1989 ; Industrial Technology Institute, 1989 ; Statistique Canada, 1988 et 1989).

\subsection{Les répondants}

Un questionnaire ayant déjà fait l'objet d'un prétest auprès de 10 personnes fut envoyé directement aux dirigeants de 366 entreprises manufacturières. Ces entreprises représentent toutes les PME actives au Québec dans le secteur de la transformation des plastiques. La plus stricte confidentialité fut assurée aux participants. Les dirigeants de 95 entreprises acceptèrent de collaborer à cette enquête, ce qui constitue un taux de réponse de $26,3 \%$. Aucun rappel ne fut effectué. Pour respecter les critères de taille préétablis, une entreprise a dû être exclue, puisque ses effectifs excédaient 200 employés.

Environ $21 \%$ des entreprises qui ont participé à cette recherche dépendent d'un siège social. Or, la présence d'un siège social est un facteur externe qui peut modifier de nombreux facteurs internes dont, en particulier, l'adoption des nouvelles technologies. Nous excluerons donc ces entreprises, ce qui porte l'échantillon à 74 entreprises que nous qualifierons d'entreprises indépendantes, tout en sachant que cette qualification est légèrement abusive (Cibert, 1990).

\subsection{Choix des mesures operationnelles des variables de recherche}

Les variables de nature factuelle qui correspondent aux caractéristiques personnelles du dirigeant ne posent guère de problèmes de mesure. Toutes les autres variables de nature perceptuelle sont mesurées à partir de construits validés. Les mesures utilisées dans cette recherche sont présentées au tableau 3.

\section{Discussion des résultats}

L'objectif fondamental de cette recherche est d'étudier la relation entre le degré innovateur et les variables présentées au tableau 3. Notons que ceci constitue une démarche assez inédite, puisque très peu de recherches empiriques ont pris 
en considération le caractère radical des innovations. Une exception notable est sans aucun doute la recherche menée en 1977 par Dewar et Dutton sur 40 entreprises dans l'industrie de la chaussure (Dewar et Dutton, 1986); l'autre exception est la recherche d'Ettlie et al. (1984). Selon nous, aucune évidence empirique n'existe sur ce plan dans le milieu spécifique des PME et des applications informatisées.

Les résultats du tableau 3 montrent clairement que les innovations plus radicales demandent par leur nature même une expertise interne très élevée. L'expérience fonctionnelle en génie production du dirigeant est positivement associée au degré innovateur, et ce, de façon significative, alors qu'une relation négative est observée au regard de l'expérience fonctionnelle en comptabilité/ finance. Notons que le nombre d'années d'études est positivement, mais non fortement associé au degré innovateur. Par contre, le nombre d'années durant lesquelles il a travaillé (qui peut être considéré comme une approximation de l'âge du dirigeant) donne des résultats peu concluants.

L'innovation apporte des changements majeurs et profonds qui exigent des attitudes très positives de la part de la haute direction, ce qui est clairement confirmé par le tableau 1. Une attitude favorable envers les changements technologiques et le risque, une attitude proactive et un champ de contrôle interne sont autant de facteurs positifs.

Les innovations plus radicales impliquent des éléments de nouveauté, de nombreuses incertitudes et certaines difficultés peu prévisibles. Ces innovations sont considérées comme potentiellement perturbatrices (disruptives) voire menaçantes (Ettlie et al., 1984). Par conséquent, il semble que leur adoption dans un contexte de PME demanderait une recherche systématique d'informations très intense, un horizon de planification qui dépasse le court terme, des analyses plus poussées et une conscience accrue de l'orientation stratégique de l'entreprise. Toutes ces prévisions sont confirmées par les résultats du tableau 1. Notons que l'information externe a moins de poids que l'information interne.

Les structures organisationnelles et leur relation avec l'adoption d'innovations ont retenu l'attention de plusieurs chercheurs (par exemple, Collins et al., 1988). Cependant, les résultats restent contradictoires en fonction du type d'innovation et du contexte organisationnel. La technocratisation, reflétant la concentration du personnel technique, aurait tendance à promouvoir des innovations incrémentales, car les efforts innovateurs deviennent alors institutionnalisés (Hage, 1980). À notre avis, cet argument ne s'applique guère à des PME mais plutôt à des entreprises de plus grande taille, comme celles étudiées par Hage. Plus récemment, Ettlie et al. (1984) ont démontré qu'une politique technologique agressive alliée à une concentration de spécialistes favoriserait les innovations plus radicales. Dans la même voie, Dewar et Dutton arrivent à la 
conclusion que la technocratisation est l'élément majeur pour l'adoption d'innovations plus radicales, ce que les résultats de cette recherche confirment de façon indéniable. Plus le nombre d'employés à caractère technique est important, plus les nouvelles idées, les nouveautés technologiques et les nouvelles procédures peuvent être comprises et mises en place.

Bien que la centralisation soit parfois positivement, parfois négativement associée à l'innovation, nous partageons l'opinion de Dewar et Dutton (1986) selon laquelle les changements profonds générés par les innovations plus radicales nécessitent une concentration du pouvoir décisionnel. Mais les innovations plus radicales demandent énormément de souplesse et d'adaptation, comme en témoigne l'expression "controlled chaos " utilisée pour illustrer ce phénomène (Quinn, 1985). Bien que la concentration du pouvoir décisionnel soit de façon générale très élevée en milieu de PME, elle ne semble pas avoir de relation avec le degré innovateur $(r=-0,04, p=0,372)$. Toutefois, l'aspect " formalisation » dans le tableau 3 laisse transparaître des différences beaucoup plus importantes, les structures plus formelles ayant une relation négative significative avec le degré innovateur.

L'analyse du tableau 3 ne pourrait être complète sans examiner l'effet de la taille. Les résultats des recherches précédentes sont contradictoires quant à l'effet de la variable " taille ». Quelques études ont démontré que les entreprises les plus petites sont les plus innovatrices (Rothwell, 1978 ; Globerman, 1975). Toutefois, l'hypothèse inverse, à savoir que le degré d'innovation est directement proportionnel à la taille, a été soutenue par les études de Moch et Morse (1977), Armour et Teece (1980) ainsi que Kimberly et Evanisko (1981). Il est possible de penser que le caractère radical d'une innovation vienne nuancer cette relation. Rothwell (1978) explique ses résultats en soulignant que ce ne seront pas les petites et moyennes entreprises qui deviendront innovatrices si cette transformation requiert d'importants investissements financiers. Par ailleurs, il semblerait que l'accroissement de la taille d'une entreprise n'est pas de nature à favoriser l'adaptation structurale propice à l'émergence de sa vocation innovatrice (Moch et Morse, 1977 ; Zaltman et al., 1973; Inkson et al., 1970). D'après le tableau 3, la relation entre la taille de l'entreprise et le degré innovateur est légèrement positive mais non significative, ce qui constitue un indice partiel selon lequel l'adoption cumulée de plusieurs innovations requiert des ressources plus importantes. Cependant, la taille semble jouer un rôle très secondaire par rapport aux attitudes et aux traits de personnalité et aux caractéristiques du processus décisionnel du dirigeant. 


\section{TABLEAU 3}

Analyse des corrélations entre le degré innovateur et les variables indépendantes $(n=74)$

\begin{tabular}{|c|c|c|}
\hline Variables indépendantes & $\begin{array}{l}\text { Coefficient } \\
\text { de Pearson }\end{array}$ & $\begin{array}{l}\text { Niveau de } \\
\text { signification (5) }\end{array}$ \\
\hline Nombre d'années de service dans l'entreprise & 0,04 & 0,374 \\
\hline Nombre d'années de service dans ce secteur & $-0,13$ & 0,128 \\
\hline $\begin{array}{l}\text { Nombre d'années de service en tant que } \\
\text { dirigeant de cette entreprise }\end{array}$ & 0,03 & 0,396 \\
\hline Expérience fonctionnelle en comptabilité/finance & $-0,32$ & $0,003 * * *$ \\
\hline $\begin{array}{l}\text { Expérience fonctionnelle dans } \\
\text { les ventes/commercialisation }{ }^{(1)}\end{array}$ & $-0,06$ & 0,318 \\
\hline Expérience fonctionnelle en génie/production & 0,44 & $0,000 * * *$ \\
\hline $\begin{array}{l}\text { Expérience fonctionnelle en gestion } \\
\text { des ressources humaines } \\
(1)\end{array}$ & $-0,12$ & 0,163 \\
\hline Années d'études ${ }^{(3)}$ & 0,18 & $0,059 *$ \\
\hline Attitude envers les changements technologiques ${ }^{(1)}$ & 0,40 & $0,001 * * *$ \\
\hline Attitude envers le risque (1) & 0,52 & $0,000 * * *$ \\
\hline Attitude proactive $(1)$ & 0,49 & $0,000 * * *$ \\
\hline Champ de contrôle (2) & $-0,39$ & $0,000 * * *$ \\
\hline Recherche systématique d'information externe ${ }^{(1)}$ & 0,34 & $0,002 * * *$ \\
\hline Recherche systématique d'information interne ${ }^{(1)}$ & 0,45 & $0,000 * * *$ \\
\hline Horizon de planification ${ }^{(1)}$ & 0,47 & $0,000 * * *$ \\
\hline Analyse ${ }^{(1)}$ & 0,47 & $0,000 * * *$ \\
\hline Conscience de la stratégie & 0,37 & $0,001 * * *$ \\
\hline Technocratisation & 0,37 & $0,001 * * *$ \\
\hline Centralisation ${ }^{(1)}$ & $-0,04$ & 0,372 \\
\hline Formalisation ${ }^{(1)}$ & $-0,35$ & $0,001 * * *$ \\
\hline Taille (ln des ventes) ${ }^{(4)}$ & 0,11 & 0,181 \\
\hline
\end{tabular}

(1) Mesures basées sur des échelles de Likert en 7 points d'ancrage où 1 indique le plus bas niveau et 7 le plus haut.

(2) Une valeur peu élevée indique un champ de contrôle inteme et une valeur élevée indique un champ de contrôle exteme.

(3) Échelle ordinale ; graduation de 0 à 5 selon les années d'études : 0 désigne les études secondaires et 5 les études de doctorat.

(4) Le logarithme naturel est ici utilisé pour normaliser cette variable.

(5) ${ }^{* * *} \mathrm{p}<0,01 ;{ }^{* *} \mathrm{p}<0,05 ;{ }^{*} \mathrm{p}<0,10$. 


\section{Conclusion}

En contexte de PME, l'étude des actions stratégiques ne peut être menée sans tenir compte a priori du caractère propre du dirigeant, puisque la direction stratégique de l'entreprise sera fortement imprégnée de son influence (Adler, 1989; Mintzberg, 1988 ; Miller et Toulouse, 1986). L'adoption de nouvelles technologies est certes l'une de ces actions dites stratégiques (Shrivastava et Grant, 1985) mais, cependant, trop peu d'études empiriques s'y consacrent en contexte de PME. À cet égard, nous croyons que notre recherche a permis de mieux cerner la contribution de l'ensemble des caractéristiques associées aux dirigeants dans l'action stratégique que représente l'adoption des nouvelles technologies informatiques.

Ainsi, les résultats de cette recherche empirique ont démontré que l'attitude envers le risque et l'attitude proactive sont les plus fortement corrélées au degré innovateur, suivies de trois caractéristiques du processus décisionnel du dirigeant (horizon de planification à long terme, méthodes d'analyses plus poussées et recherche systématique d'informations internes). L'expérience fonctionnelle en génie-production serait aussi un atout qui favorise l'adoption des nouvelles technologies. On peut aussi constater une primauté des variables individuelles (reliées à l'individu, c'est-à-dire au dirigeant) sur les trois variables organisationnelles (reliées à l'organisation) considérées. Ceci suggère que si les différences individuelles ne sont pas prises en compte dans l'étude des facteurs d'adoption des nouvelles technologies, certaines variables primordiales risquent fort d'être négligées, du moins en contexte de PME.

Enfin, la redéfinition proposée du degré innovateur permet une graduation plus subtile en considérant non seulement le nombre d'innovations mais aussi leur attribut secondaire, soit leur nature radicale ou incrementale. Le score ainsi obtenu permet de mieux classifier les PME selon un continuum de moins innovatrices à plus innovatrices et pallie certaines difficultés méthodologiques énoncées en particulier par Downs et Mohr.

En résumé, ces résultats s'inscrivent dans la volonté de mieux cerner et de mieux définir la problématique de l'adoption et de la diffusion des nouvelles technologies dans nos PME manufacturières. À cet égard, il est essentiel de relever les différentes dimensions du rôle des dirigeants lors de l'adoption de ces technologies. Alors peut-être pourrons-nous mieux comprendre un des facteurs essentiels à l'adoption des nouvelles technologies et ultimement élaborer à la fois des options politiques et des programmes de formation susceptibles de supporter et de promouvoir les efforts accordés à l'impératif technologique. 


\section{Bibliographie}

ADLER, P.S. (1989), «Technology strategy : a guide to the literatures » dans R.S. Rosembloom and R.A. Burgelman (éd.), Research on Technological Innovation, Management and Policy, Londres, JA Press Inc., vol. 4, p. 25-151.

ARMOUR, H.O. et D.J. TEECE (1980), « Vertical integration and technological innovation ", Review of Economics and Statistics, vol. 62, p. 470-474.

Association CAO/FAO (1989), « Tendances et degré de pénétration : l'automatisation et l'informatisation de la production », gouvernement du Québec.

BaRAnson, J. (1981), The Japanese Challenge to U.S. Industry, Lexington, Massachusetts, Lexington Books.

BrockHAUs, R.H. (1982), Encyclopedia of Entrepreneurship, New Jersey, Prentice-Hall.

BROCKHAUS, R.H. (1975), «I.E. locus of control scores as predictors of entrepreneurial intentions », Proceedings of Academy Management, août, p. 433-435.

BRockHAUs, R.H. (1980), «Risk taking propensity of entrepreneurs », Academy of Management Journal, vol. 23, p. 509-520.

CAsTaldi, R.M. (1986), "An analysis of the work roles of CEOs of small firms ", American Journal of Small Business, été, p. 53-64.

CEC (Conseil économique du Canada) (1986), La technologie en milieu de travail : enquête sur l'automatisation au Canada, Ottawa.

CEC (Conseil économique du Canada) (1987), Le recentrage technologique, $n^{\circ}$ de catalogue EC22-142/1987 F, Ottawa.

CrIIL, J. et al. (1983), «A price to pay? Professionalism and work organization in Britain and West Germany », Sociology, vol. 17, $n^{\circ} 1$, p. 63-78.

CiBerT, A. (1990), «Perspectives », Revue Internationale PME, vol. 3, n 2, p. 139-145.

CoHN, S.F. et R.M. TURYN (1984), « Organizational structure, decision-making procedures and the adoption of innovations », IEEE Trans-Engineering Management, EM. 31, p. 154-161.

Coluns, P.D., J. HAGE et F.M. Hull (1988), « Organizational and technological predictors of change in automaticity », Academy of Management Journal, vol. $31, \mathrm{n}^{\circ} 3$, p. 512-543.

D'AmBoise, G. (1989), La PME canadienne : situation et défis, L'Institut de recherches politiques, Québec, Les Presses de l'Université Laval.

DEAN, J.W. (1987), «Building the future : the justification process for new technology », dans New Technology as Organizational Innovation, Ballinger Publishing Company.

DEWAR, R.D. et J.E. DutTon (1986), «The adoption of radical and incremental innovations : an empirical analysis », Management Science, vol. 32, $\mathrm{n}^{\circ} 11, \mathrm{p} .1422-1433$.

Downs, G.W. et L.B. Morr (1976), «Conceptual issues in the study of innovation », Administrative Science Quarterly, vol. 21, p. 700-714.

DURAND, D.E. et D. SHEA (1974), «Entrepreneurial activity as a function of achievement motivation and reinforcement control », Journal of Psychology, vol. 88, p. 57-63.

ETTLIE, J.E., W.P., BRIDGES et R.D. O'KeEFE (1984), « Organization strategy and structural differences for radical versus incremental innovation », Management Science, vol. $30, n^{\circ} 6$, p. 682-695. 
Gerstein, M. et H. Reisman (1984), « Creating competitive advantage with computer technology », The Journal of Business Strategy, vol. 3, $n^{\circ} 1$, p. 53-60.

Gibson, C. et R. Nolan (1974), «Managing the four stages of EDP growth », Harvard Business Review, vol. 52, janvier-février, p. 76-88.

Globerman, S. (1975), « Technological diffusion in the Canadian carpet industry », Research Policy, 4, p. 129-148.

GolDHAR, J.D. et M. JeLINEK (1985), «Computer integrated flexible manufacturing : organizational, economic and strategic implications », Interfaces, vol. 15, $\mathrm{n}^{\circ} 3$, p. 94-105.

HAGE, J. et M. AIKen (1967), « Program change and organizational properties : a comparative analysis ", American Journal of Psychology, vol. 72, p. 503-519.

HAGe, J. et M. AIKen (1970), Social Change in Complex Organizations, Random House, New York.

HARVARD BusINess Review (1987), « Competitiveness survey: HBR readers respond ", septembre-octobre, p. 8-12.

HAYEs, R. (1981), «Why Japanese factories work », Harvard Business Review, juilletaoût, p. 56-66.

HAYES, R.H. et S.C. WheElWright (1984), Restoring our Competitive Edge : Competing Through Manufacturing, John Wiley.

Hayes, R.H. et W.J. AbeRnATHY (1980), «Managing our way to economic decline », Harvard Business Review, juillet-août, p. 67-77.

Industrial TechNology Institute (1989), «Technology patterns and applications », Frostbelt Automation, vol. 1.

Inkson, J.H.K., D.S. PugH et D.J. HICKson (1970), « Organization content and structure : an abbreviated replication », Administrative Science Quarterly, vol. 15, p. 318-29.

JARYMISZYN, P., K.B. ClARK et L.H. SumMERS (1985), « Chief executive background and firm performance ", dans K.B. Clark et al. (éd.), The Uneasy Alliance : Managing the Productivity - Technology Dilemna, éditeurs : Clark, Cambridge, Harvard Business School Press.

Julien, P.A. et M. Marchesnay (1988), La petite entreprise, Paris, Librairie Vuibert.

Julien, P.A., J.B. CARRİere, et L. HÉBERT (1988), « La diffusion des nouvelles technologies dans trois secteurs industriels », Conseil de la science et de la technologie, Québec, document $\mathrm{n}^{\circ}$ 88-03.

KANTROW, A.M. (1980), « The strategy/technology connection », Harvard Business Review, vol. 53, $\mathrm{n}^{\circ} 4$.

Kets De VRIES, M. et D. Miller (1984), The Neurotic Organization, San Francisco, Jossey-Bass,

Khan, A.M. et V. Manopichetwattana (1989), «Innovative and non-innovative small firms : types and characteristics », Management Science, vol. 35, $n^{\circ} 5$, p. 597-606.

Khandwalla, P.N. (1977), The Design of Organizations, New York, Harcourt Brace Jovanovich Inc.

KIMBERLY, J.R. et M.J. EvaNISKo (1981), « Organizational innovation : the influence of individual, organizational and contextual factors on hospital adoption of technological and administrative innovations », Academy of Management Journal, vol. 24 , p. $689-713$. 
Lawrence, P. (1980), Managers and Management in West Germany, New York, St-Martin's Press.

LeFeBvre, É., L.A. LeFeBVRE, et D. Colin (1990), « Facteurs d'adoption des nouvelles technologies de production dans les PME manufacturières innovatrices : une perspective évolutive ", Revue internationale $P M E$, vol. 3, $\mathrm{n}^{\circ}$ 2, p. 215-229.

LEFEBVRE, L.A. et É. LeFeBVRE (1988a), «The innovative business firm in Canada : an empirical study of CAD-CAM firms ", International Labour Review, vol. 127, $n^{\circ} 4$, p. 497-513.

LeFEBVRE, L.A. et É. LeFeBVre (1988b), « Computerization of small firms - a study of the perceptions and expectations of managers », Journal of Small Business and Entrepreneurship, vol. 5, $\mathrm{n}^{\circ} 5$.

LeFEBVRe, L.A., É. LeFeBvre et J. DuchaRme (1985), «Les entreprises québécoises : situation actuelle et perspectives futures $»$, Revue internationale de gestion, vol. $10, n^{\circ} 4$, p. $31-34$.

LIMPRECHT et R. HAYES (1982), «Germany's world class manufacturers », Harvard Business Review, novembre-décembre, p. 137-145.

LUMPKIN, J.R. (1988), "Establishing the validity of an abbreviated locus of control scale : is a brief levenson's scale any better? », Psychological Reports, vol. 63, p. 519-523.

MAIDIQUE, M.A. et R.H. HAYES (1985), « The art of high technology management », dans Managing Change and Uncertainty, vol. $25, \mathrm{n}^{\circ} 2$, Sloan Management Review Association, p. 17-31.

MARSLAND, S. et M. BEeR (1983), « The evolution of Japanese management : lessons for U.S. managers », Organizational Dynamics, hiver, p. 49-67.

MCMILLAN, C.J. (1987), «The automation triangle : new paths to productivity performance », Business Quarterly, automne, p. 61-67.

MeYER, A.D. et J.B. Goes (1987), « How organizations adopt and implement new technologies ", Forty-seventh annual meeting of the Academy of management, New Orleans, p. 175-179.

MIC (Ministère de l'Industrie et du Commerce) (1987), « Les PME au Québec : état de la situation ", Rapport du ministre délégué aux PME, gouvernement du Québec.

MILler, D. et C. Droge (1986), «Traditional and psychological determinants of organization structure », Administrative Science Quarterly, vol. 31, p. 539-560.

MIILER, D. et J.M. Toulouse (1986a), «Chief executive personality and corporate strategy and structure in small firms ", Management Science, vol. 32, $\mathrm{n}^{\circ} 11$, p. 1389-1409.

MrLLeR, D. et J.M. Toulouse (1986b), « Strategy, structure, CEO personality and performance in small firms ", American Journal of Small Business, hiver, p. 47-62.

Miller, D., M.F.R. KeTS DE VRIES et J.M. Toulouse, (1982), « Top executive locus of control and its relationship to strategy-making, structure and environment », Academy of Management Journal, vol. 25, $\mathrm{n}^{\circ}$ 2, p. 237-253.

MinTZBERG, H. (1988), « Strategy-making in three modes » dans J.B. Quinn, H. Mintzberg and R.J. James (éd.), The Strategy Process, New Jersey, Englewood Cliffs, Prentice Hall, p. 82-89.

Moch, M.K. et E.T. MoRse (1977), « Size, centralization and organizational adoption of innovations », American Sociological Review, vol. 42, p. 716-725. 
MunRo, H. et H. Noori, (1987), « Canada and new technology, part I : Canada's international performance », National centre for management research and development, Western University, cahier de recherche $n^{\circ}$ NC87-10.

NABSETH, L. (1973), "The diffusion of innovation in Sweedish industry », dans B.R. Williams (éd.), Science and Technology in Economy Growth, Londres, McMillan Press.

NoRmanN, R. (1971), «Organizational innovativeness : product variation and reorientation », Administrative Science Quarterly, vol. 16, p. 203-215.

Pennings, J.M. (1987), New technology as organizational innovation, J.M. Pennings et A. Buitendam (éd.), Ballinger Publishing Company.

QuINN, J.B. (1985), « Managing innovation: controlled chaos », Harvard Business Review, mai-juin, p. 73-84.

RAYMOND, L. (1987), "An empirical study of management information systems sophistication in small business », Journal of Small Business \& Entrepreneurship, été, p. 38-47.

Rogers, E.M. et F. Shoemaker (1971), Communications of Innovations, New York, The Free Press.

Rothwel, R. (1978), « Small and medium sized manufacturing firms and technological innovation ", Management Decision, vol. 16, n 6, p. 362-369.

ROTTER, J.B. (1966), «Generalized expectations for internal versus external control of reinforcement », Psychological Monographs : General and Applied, vol. 80, $\mathrm{n}^{\circ} 1$.

SCHONBERGER, R.J. (1982), Japanese Manufacturing Techniques, New York, Free Press.

Shapero, A. (1975), « The displaced, uncomfortable entrepreneur », Psychology Today, vol. $9, n^{\circ} 6$, p. 83-88.

Shrivastava, P. et J.H. Grant (1985), « Empirically derived models of strategic decisionmaking processes », Strategic Management Journal, vol. 6, p. 97-113.

Simon, H.A. (1977), The New Science of Management Decision, Englewood Cliffs, Prentice Hall.

SkINNER, W. (1985), Manufacturing : The Formidable Competitive Weapon, New York, John Wiley.

SKINNER, W. (1984), "Operations technology : blind spot in strategic management », Interfaces, vol. $14, \mathrm{n}^{\circ} 1, \mathrm{p} .116-125$.

SKINNER, W. (1983), «Wanted, managers for the factory of the future ", Annals of the American Academy of Political and Social Science, vol. 470, p. 102-114.

Statistique Canada (1988), «Enquête sur la technologie de la fabrication », $n^{\circ} 88-03-$ 15.

Statistique Canada (1989), "Survey of manufacturing technologies ", Statistical tables, science technology and capital stock division.

Zaltman, G., R. Duncan et J. HolbeK (1973), Innovations and Organizations, New York, John Wiley. 